
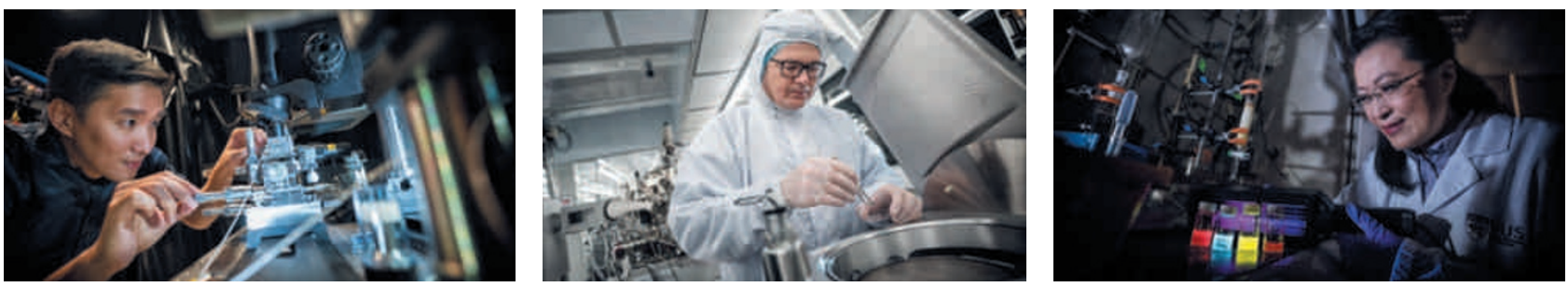

\title{
National University of Singapore
}

\section{Developing Asian-inspired solutions to global problems}

The National University of Singapore is becoming an attractive launch pad for postdoctoral researchers, who are drawn by world-class laboratories, ground-breaking science and extensive links to government, academia and industry both in Singapore and globally.

$\mathrm{n}$ recent years, the National University of Singapore (NUS) has consistently come up tops in the regional and global assessments of academic institutions. The Times Higher Education World University Rankings 2016 ranked NUS first in Asia and among the top 30 universities in the world. And the 2016 Nature Index places it among the world's top 50 research institutions.

These rankings are recognition of the university's ongoing accomplishments in education and research. NUS' achievements have made it a hub for postdoctoral researchers hoping to take their science to a new level. The opportunity to perform high-quality research in a well-connected, interdisciplinary environment with some of the world's best facilities and science leaders makes NUS a highly attractive launch pad for a successful research career. Adding to this, NUS' location in the midst of a rapidly transforming Asia offers the chance to be at the forefront of new discovery as the focus of research in fields such as health and the environment intensifies in the region.

The 111-year-old institution began as a medical school with 23 students in 1905, but it has gone on to become Singapore's premier research-intensive university with more than 3,300 researchers working in 17 schools and faculties. In addition, there are 27 university-level research institutes and centres, as well as three prestigious national Research Centres of Excellence (RCE) on campus, with NUS being a key partner in Singapore's fifth RCE.
Responding to the environmental, economic, health and social needs of Asia and the world, the university's areas of focus include materials science, integrative sustainability solutions, biomedical science, translational medicine and ageing. The highly organized and integrated nature of the island-state means that NUS researchers working in these and other areas can directly impact government policy, translating their research into real-world impact.

And while medicine continues to play a central role in the institution's research portfolio, the university also has teams pushing the boundaries of quantum physics, nanotechnology, chemical and biomolecular engineering, water and environmental research, and other fields.

\section{Space entanglement}

On 16 December 2015, tensions were high among one of those teams at the NUS Centre for Quantum Technologies, an RCE.

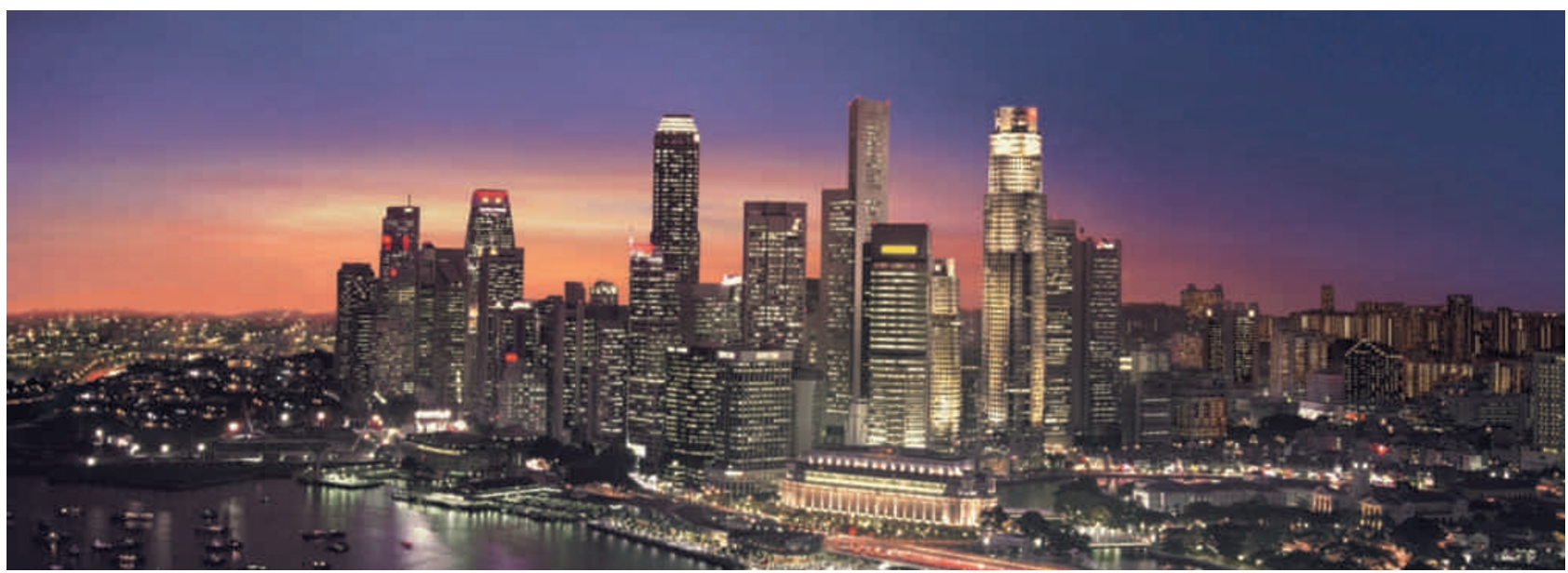


Assistant Professor Alexander Ling (top left image on adjacent page) and his research group of 12 , including five postdoctoral researchers and four PhD students, were waiting for the first data back from Galassia - an NUS-built experimental cube-satellite weighing about two kilograms - on which their quantum communication and computing device, called Small Photon Entangling Quantum System (SPEQS), had been propelled into orbit. It was a worldfirst attempt to see whether quantum technology functions in space - an early step in a much larger project to develop a highly secure quantum internet.

"The experiment was a success, and plans are already underway for further trials of the equipment," says Ling. "Our aim is to establish space-to-ground links from satellites in low Earth orbit for quantum key distribution, a technique for secure communication. Having space-to-ground links will enable the development of a global network for distributing quantum entanglement." Ling's multinational team is one of the pioneering groups to use nanosatellites for performing fundamental science experiments in space.

In addition to being a multinational team, the work requires Ling's lab to be multidisciplinary - bringing together a combination of deep scientific knowledge and instrumentation skills. Specialists in experimental quantum physics as well as mechanical and electrical engineering are working alongside each other towards a common goal. "Every single person in my team learns new things all the time, but this synthesis allows us to develop the solutions that are necessary for the instruments to operate in space."

Back on Earth, strong government support means that NUS offers some of the world's most sophisticated equipment and facilities, including the Singapore Synchrotron Light Source and the Centre for Ion Beam Applications.

In 2014, the Centre for Advanced 2D Materials was established with S\$50 million funding over 10 years from Singapore's National Research Foundation. The funding is helping to run laboratories and micro- and nanofabrication facilities used to explore and synthesize two-dimensional materials such as graphene, as well as to develop new devices based on these exceptional materials. "There are very few places in the world that offer similar access to the tools optimized for research into graphene and two-dimensional materials," says Assistant Professor Slavan Garaj (top centre image on adjacent page), leader of the centre's Nano/Bio Physics Laboratory.

One of the benefits of having different research groups and disciplines working in these facilities is what Garaj calls the cluster effect. "Our community of likeminded researchers tackling different problems with the same tools leads to free permeation of practical know-how."

His team includes seven postdoctoral researchers from five countries and among them are physicists, materials scientists, chemists and biotechnologists. "They are constantly learning from each other," says Garaj. "The work offers an experience of nanoscience at its finest, with different scientific disciplines fused together."

Similar to other NUS lab leaders, Garaj pays particular attention to the postdoctoral members of his team. "My firm belief is that my laboratory is only as successful as its alumni," he says. He takes time to discuss their specific research career goals, works with them to adjust their projects in order to better achieve those goals, and helps to position them well within research and industrial collaborations.

\section{Crossing boundaries}

Collaboration and interdisciplinary connections are an important facet of all major research activities at NUS. Some of these are housed on campus, such as those with the Massachusetts Institute of Technology and ETH Zurich. There are also important partnerships with top research institutions from around the world, including Harvard University and Stanford University. The newly formed Global Alliance between NUS, the University of Cambridge and University of California, Berkeley, will soon see the establishment of joint research programmes shared by the three universities.

Connections with industry also play an important role in research translation, commercialization and entrepreneurship. Professor Liu Bin (top right image on

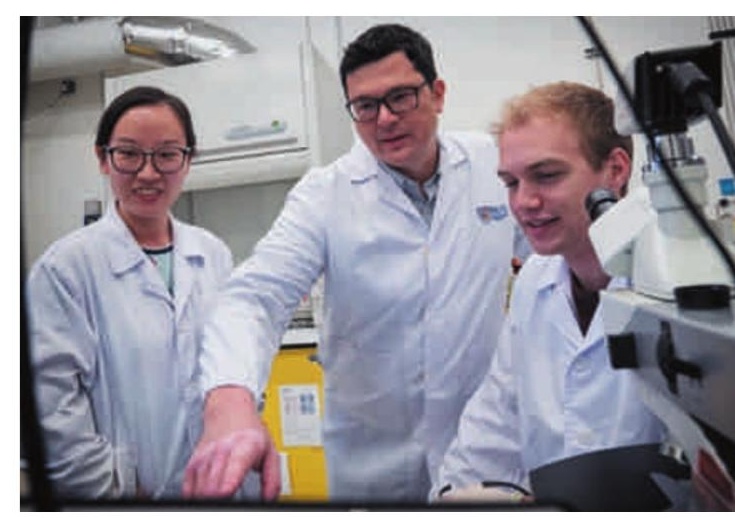

adjacent page), a chemist, has been working with the healthcare industry and has co-founded the spin-off company LuminiCell to develop and commercialize her work at the forefront of cell luminescence. "The opportunities and schemes to encourage collaboration between academia and industry are tremendous in Singapore," says Liu, who is acclaimed as one of the world's most influential minds in science.

Work by Liu's group in the Department of Chemical and Biomolecular Engineering at the NUS Faculty of Engineering looks at the design and synthesis of polymers with optimized light harvesting and small molecules with aggregationinduced emission, with a particular focus on water-soluble materials. "Our research has a direct impact on everyday life, from light-emitting devices to healthcare products," she says.

And just as Liu's work in cell luminescence enables us to shine a light in hard-to-reach areas - more effectively tracking diseases and transmitting communications - postdoctoral research at NUS provides an exciting podium for some of the best emerging scientific talents to spotlight the research questions of their choice with the finest facilities, the support of global science leaders and the opportunity for multidisciplinary collaboration on and off campus.

JP276347R

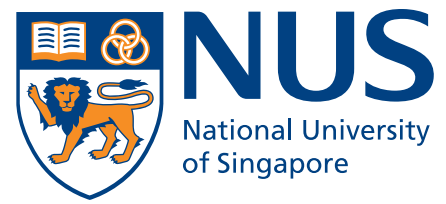

Website:

nus.edu.sg 


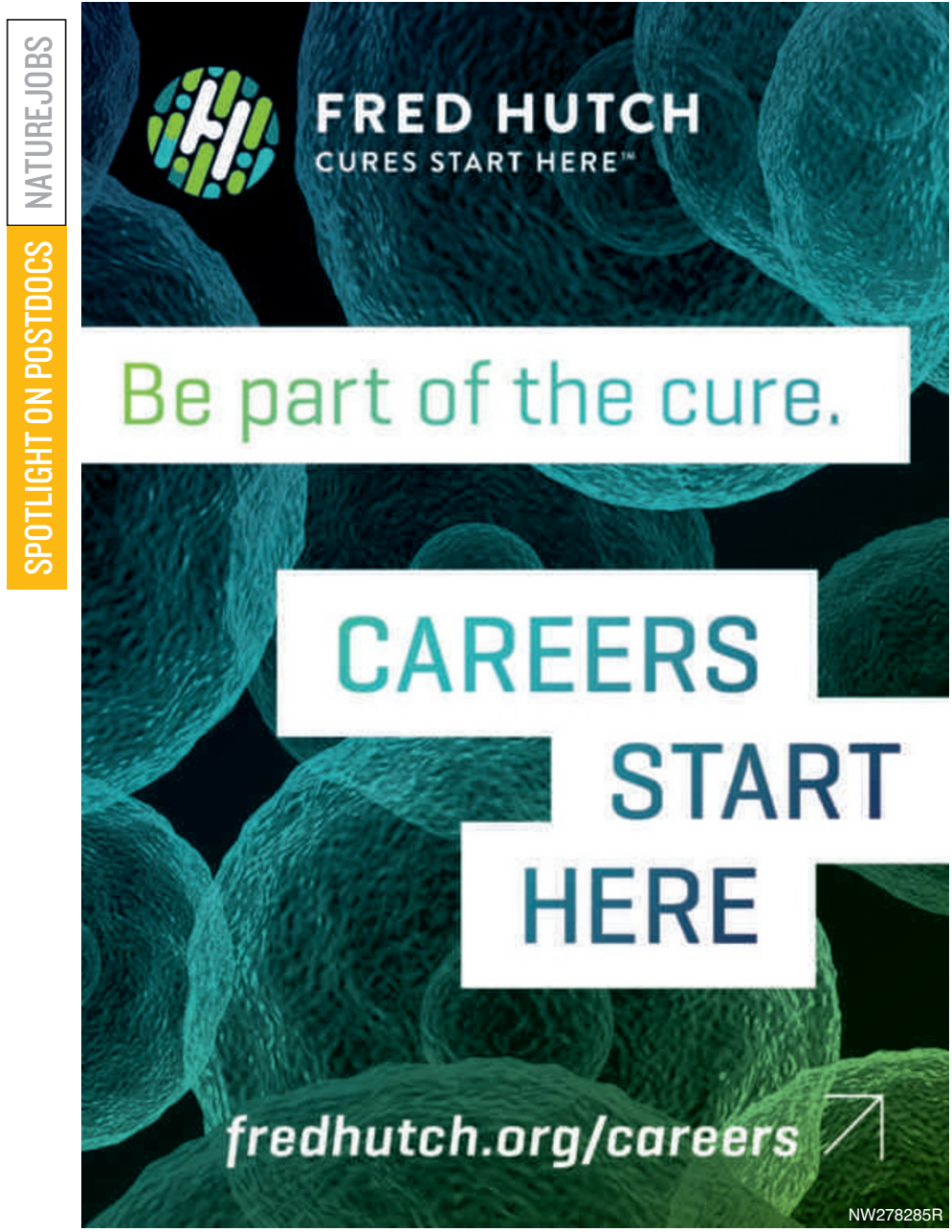

\section{Lerner Research Institute Postdoctoral Fellowships}

Cleveland Clinic, a top two hospital in the United States by US News and World Report, is home to the Lerner Research Institute (LRI), consistently ranked in the top 10 of $\mathrm{NIH}$ funded research institutes in the US. A hallmark of the LRI is a focus on disease-oriented and high-impact research, representing collaborative interactions between scientists working in basic research laboratories and multiple clinical specialties. Major research areas include cardiovascular disease (Zhu, et al., Gut Microbial Metabolite TMAO Enhances Platelet Hyperreactivity and Thrombosis Risk 2016 Cell 165:111-24; Chen, et al., PCSK6-Mediated Corin Activation is Essential for Normal Blood Pressure, 2015 Nat Med.21:1048-53), cancer (Li, et al., Conversion of Abiraterone to D4A Drives Anti-Tumour Activity in Prostate Cancer, 2015 Nature 523:347-51; Schonberg, et al., Preferential Iron Trafficking Characterizes Glioblastoma Stem-like Cells, 2015 Cancer Cell 28:441-55; Eswarappa, et al., Programmed Translational Readthrough Generates Antiangiogenic VEGF-Ax, 2014 Cell 157:1605- 8), and autoimmune and inflammatory disease (Kang Z, et al, Act1 mediates IL-17-induced EAE pathogenesis selectively in NG2+ glial cells. Nat Neurosci, 2013 16:1401-8; Martin BN, IKKalpha negatively regulates ASC-dependent inflammasome activation, 2014 Nat Commun 5:4977).

Postdoctoral opportunities are available for highly motivated individuals in multiple laboratories. Specific fellowship descriptions are available at http://www.lerner.ccf.org/jobs/postdoctoral/

PRINCETON
UNIVERSITY
Princeton University
Department of Chemistry
Assistant Professor
The Department of Chemistry at Princeton
University invites applications for a tenure-track
assistant professor position. We are especially
interested in candidates working in the areas of
experimental physical chemistry or inorganic
chemistry. We seek a faculty member who will
create a climate that embraces excellence and
diversity with a strong commitment to research
and teaching that will enhance the work of the
department and attract and retain a diverse
student body. We strongly encourage
applications from members of all
underrepresented groups. Candidates are
expected to have completed the Ph.D. in
chemistry or a related field at the time of
appointment. Applicants should submit a
description of research interests, curriculum
vitae, a list of publications, and contact
information for three referees online at
jobs.princeton.edu/applicants/Central?quickFind
=68816. For fullest consideration, candidates
are encouraged to submit completed
applications by October 15,2016 .
Princeton University is an equal opportunity
employer. All qualified applicants will receive
consideration for employment without regard to
race, color, religion, sex, national origin, disability
status, protected veteran status, or any other
characteristic protected by law. This position is
subject to the University's background check policy.
ant

NW280718R

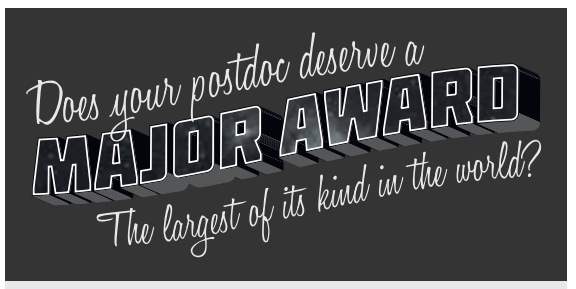

Nominate them for

The Origins Project

Postdoc Lectureship Award

This annual $\$ 10,000$ international award, the largest of its kind in the world, will be given to an outstanding junior scholar chosen from any field of study relevant to the Origins Project mission. The winner will be hosted at ASU in Tempe, Arizona for one week, and will present 3 lectures on their research. In addition to the cash award, all travel and accommodation expenses will be covered by the Origins Project. NW280363R

origins.asu.edu/postdocaward Nomination deadline Nov. 1, 2016

AST Origins

\section{(事) \\ Memorial Sloan Ketterin CincerCenter}

FACULTY POSITIONS FOR PHYSICIAN SCIENTISTS IN IMMUNO-ONCOLOGY

The Cancer Immunotherapy Center at Memorial SloanKettering (MSK) invites applications for tenure track faculty appointments at the level of Assistant, Associate, or Full Member. This Center is assembling outstanding physician-scientists who wish to address problems in immunotherapy at the laboratory/clinical interface in an environment that encourages collaborative team science. Successful candidates must demonstrate the ability to develop an independent research program as well as an interest in translational oncology. Candidates will join a faculty with a broad range of research interests, including transplantation, T and NK cell development and function, gene regulation, antigen presentation, infectious disease and tumor immunology. Faculty will be housed in state-of-the-art laboratories in the new Zuckerman Research Center and jointly appointed in the Department of his/her appropriate clinical specialty at MSKCC. Faculty will also be eligible to hold appointments in the newly established Gerstner SloanKettering Graduate School of Biomedical Sciences as well as the Weill Medical School and Graduate School of Medical Sciences at Cornell University.

MSKCC offers a unique and vibrant research environment with programs in Human Oncology \& Pathogenesis, Immunology, Pharmacology, Chemistry, Molecular Biology, Computational Biology, Genetics, Cell Biology, Developmental Biology, Cellular Biochemistry, and Structural Biology and close links with the Rockefeller and Cornell communities. The presence of world-renowned clinical programs in cancer research, treatment, and prevention offers unique opportunities for creative collaboration. Applicants must have an M.D., postdoctoral experience, and an active clinical interest.

Please send curriculum vitae, a summary of current and proposed research programs, and arrange for three letters of recommendation to be sent to: Jedd Wolchok, MD, PhD and Marcel van den Brink, MD, PhD c/o Maria Alexander, Memorial Sloan-Kettering Cancer Center, 1275 York Avenue Mailbox \#340, New York, NY 10065; E-mail: facultyrecruit.mskcc@gmail.com. Memorial SloanKettering Cancer Center is an affirmative action, equal opportunity employer. 
The Cluster of Excellence CellNetworks at Heidelberg University is a world-renowned interdisciplinary research association in the and unites more than 100 internationally leading scientists from six different faculties and five non-university research centers in one common objective: to about the cells' network structure, dynamics and regulation. Founded in 2006 as part of the German Excellence Initiative, the Cluster brings together experimental life sciences, chemistry and nanotechnology as well as computer sciences and scientific computing to lift this field of research to another level.

As of 2017, CellNetworks will be offering 10 new postdoc positions to outstanding scientists. Applicants need to define their own topic of research with one or two CellNetworks member labs. Accepted fellows will be provided a two-year contract including salary and research costs as well as an annual grant of $€ 10,000$ for travelling and special demands. Deadline for all applications is mid-October 2016.

More information and the online application are available on our website at www.cellnetworks.uni-hd.de.

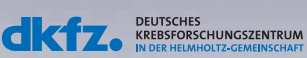
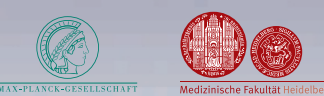

MEDIZINISCHE

FAKULTÄTMANNHEIM

DERUNIVERSTÄT

HEIDELBERG

\section{BE PART OF THE FUTURE}

$\rightarrow$ we are hiring at www.oxstem.co.uk/careers

OxStem is a pioneering drug-discovery company developing stem and progenitor cell programming therapies. Our unique approach to regenerative medicine will deliver small molecule therapeutics that enhance repair mechanisms that already exist within the body.

We are funding multiple positions within the University of Oxford and are looking to hire exceptional:

- Organic/Medicinal Chemists

- Biological and Biomedical Scientists

...to pursue R\&D programs in Oncology, Neuroscience, Ocular and Cardiac diseases as part of a fast-paced, dynamic biotechnology environment.

Bettering human health by directly impacting the quality and duration of life.

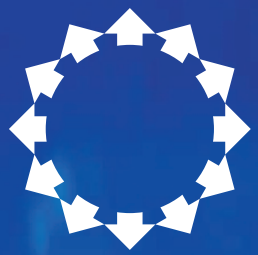

OXSTEM

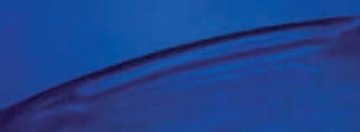

